\title{
Apoptosis: a pivotal event or an epiphenomenon in the pathophysiology of heart failure?
}

Apoptosis, or programmed cell death, is an evolutionary conserved genetically programmed process by which multicellular organisms regulate cell numbers. It is critical in development and in tissue homeostasis. Unlike necrosis, the process is active, energy requiring and precisely regulated.

\section{Apoptosis in cardiac development and disease}

Cardiac myocytes are by and large terminally differentiated and have a limited capacity for self renewal. It would therefore seem that loss of a significant number of cardiomyocytes would have lasting adverse consequences. Apoptosis is, however, integral to normal cardiac development and is also important in the morphogenesis of the conducting system. This can be illustrated by accessory pathway mediated arrhythmias caused by inadequate removal of loop short circuit cells by apoptosis. Apoptotic cardiomyocytes have also been identified in subjects with ischaemic heart disease and myocarditis. Moreover, evidence exists to show that apoptosis occurs in "end stage" heart failure. The relevance of this process in the overall pathophysiology and progression of heart failure is, however, still controversial. We hope to convey the current understanding of apoptosis with respect to its prevalence and its putative impact on chronic stable cardiomyopathy. We will also discuss the theoretical implications of blocking the apoptotic programme in the management of heart failure.

\section{Apoptosis and the pathophysiology of heart failure} Quantifying the number of apoptotic cells has been fraught with difficulty and the merits of the various analytical techniques for determining apoptosis are vigorously debated. ${ }^{12}$ Notwithstanding these limitations, recent studies demonstrate an incidence of approximately $0.2 \%$ of cardiomyocytes with apoptosis at the time of cardiac transplantation. ${ }^{34}$ Using this incidence, Feuerstein and colleagues calculated that approximately $25 \%$ of total muscle mass would be lost on an annual basis. ${ }^{5}$ This amount of cardiomyocyte loss may be feasible in patients with preterminal heart failure, but incompatible with the life expectancy of patients with chronic stable heart failure. ${ }^{6}$ We therefore hypothesise that the number of cardiomyocytes undergoing apoptosis in stable heart failure is considerably lower and of lesser significance than that reported in advanced pretransplant heart failure.

\footnotetext{
Anti-apoptotic effects of current heart failure treatment

With the improved outcome in patients with stable cardiomyopathy, ${ }^{7}$ a question arising is whether the survival promoting drugs used in the treatment of heart failure, in effect, retard the onset of apoptosis? To explore this, the concept of susceptibility to apoptosis as described by Depre and Taegtmeyer is useful to consider. ${ }^{8}$ These investigators described how a drift from cardiac contractile and energetic homeostasis confers greater vulnerability of the failing heart to apoptotic signals such as ischaemia,
}

pressure-overload, tumour necrosis factor $\alpha(\mathrm{TNF} \alpha)$, reactive oxygen species, and excess nitric oxide. In this regard angiotensin converting enzyme inhibitors and $\beta$ adrenergic antagonists have been shown, in experimental conditions, to promote resistance to apoptotic signals in the heart. ${ }^{910}$ These cell survival promoting effects may explain part of the protective mechanism of these agents in heart failure.

Pentoxifylline as an anti-apoptotic agent?

Accordingly, a study in this issue by Skudicky and colleagues is intriguing. ${ }^{11}$ They demonstrate a significant reduction in two apoptotic mediators ( $\mathrm{TNF} \alpha$ and Fas) and an improvement in contractile function in a group of patients with idiopathic cardiomyopathy treated with pentoxifylline. In this six month study, the matched control group had no deterioration in their cardiac contractile function. These data suggest that pentoxifylline may, in part, restore the contractile energetic homeostatic balance in the heart. Contractile function may improve either through the reduction in $\mathrm{TNF} \alpha$ mediated cardiodepression, ${ }^{12}$ and/or via increased intracellular cyclic adenosine monophosphate through phosphodiesterase inhibition by pentoxifylline in the treated group. ${ }^{13}$ However, based on the fact that there is a low prevalence of apoptosis in stable heart failure, and that attenuating apoptosis is unlikely to improve contractile function, the positive effects on contractile function of pentoxifylline are probably not caused by an anti-apoptotic effect despite the correlation with the plasma concentrations of Fas. Moreover, if the contractile improvement is caused by phosphodiesterase inhibition, this effect may be, in the longer term, detrimental to mortality as has been shown with other phosphodiesterase inhibitors. However, as $\mathrm{TNF} \alpha$ depresses contractile function in the stable heart failure patient and may augment apoptosis in the preterminal patient, the current studies on the direct effect of $\mathrm{TNF} \alpha$ inhibition in heart failure are eagerly awaited. ${ }^{14}$

\section{Skeletal muscle apoptosis in heart failure}

A second article in this issue by Vescove and colleagues elegantly describes an increased incidence of apoptosis in skeletal muscles of subjects with heart failure. ${ }^{15}$ Here the incidence of apoptosis is inversely correlated with peak $\mathrm{VO}_{2}$ and is postulated to be an important process in limited exercise capacity in these subjects. Moreover, the authors postulate that this apoptosis may be triggered by circulating $\mathrm{TNF} \alpha$. As the patient numbers are small in this study, it would be of interest to evaluate whether heart failure subjects treated with $\mathrm{TNF} \alpha$ inhibitors demonstrate an improvement in peak $\mathrm{VO}_{2}$.

Direct anti-apoptotic treatment in the management of heart failure?

An important question is whether directly blocking the apoptotic programme is a feasible strategy in heart failure treatment. The hypothetical risks of directly blocking apoptosis include an increased risk of neoplastic and 
autoimmune diseases. ${ }^{16}$ Thus, it is unlikely to be of overall benefit in the treatment of chronic diseases such as heart failure. Also no data exist which can determine when and if apoptosis begins to play an important role in deterioration of contractile function in subjects with chronic stable heart failure. In contrast, data are emerging which suggest that the heart can exhibit pronounced plasticity and can show improved contractile function in end stage heart failure - for example, when the heart is unloaded using the left ventricular assist device. ${ }^{17}$ Finally, additional data which support an ability to sustain life, even with a very poor left ventricular contractile function, has been shown in the Canadian implantable defibrillator study. ${ }^{18}$ These last two studies, albeit in small numbers of patients, contradict the concept that apoptosis may be a central component to the heart failure syndrome, at least in the absence of ongoing pathologic insults.

In conclusion, we believe that the current literature supports the presence of apoptosis during heart failure. However, the process itself is probably most prevalent in the premorbid heart and of limited clinical significance in the progression of heart failure in the absence of ongoing pathological insults. Furthermore, because of the theoretical risks of blocking apoptosis directly, direct anti-apoptotic agents are unlikely candidates for heart failure treatment in the near future. Strategies to diminish ongoing insult to the heart, and treatments to maintain contractile energetic homeostasis, with the resultant elevation of the apoptotic threshold, would probably have greater merit in our continuous efforts to improve the lives of patients with heart failure.

DAVID MARSHALL MICHAEL N SACK

Hatter Institute for Cardiology Research, UCT Medical School,

Cape Town 7925,

South Africa

sack@samiot.uct.ac.za
DM, is a senior clinical research fellow supported by the South African MRC. MNS is chair designate of the Roche Chair of Cellular and Preventative Cardiology at the University of Cape Town.

1 Schaper J, Elsasser A, Kostin S. The role of cell death in heart failure. Circ Res 1999;85:867-9.

2 Anversa P. Myocyte death in the pathological heart. Circ Res 2000;86:1214.

3 Olivetti G, Abbi R, Quaini F, et al. Apoptosis in the failing human heart. $N$ Engl f Med 1997;336:1131-41.

4 Guerra S, Leri A, Wang X, et al. Myocyte death in the failing human heart is gender dependent. Circ Res 1999;85:856-66.

5 Feuerstein G, Ruffolo RR, Yue T. Apoptosis and congestive heart failure. Trends in Cardiovascular Medicine. 1997;7:249-55.

6 Felker GM, Thompson RE, Hare JM, et al. Underlying causes and long-term survival in patients with initially unexplained cardiomyopathy. $N$ long-term survival in patients with
Engl f Med 2000;342:1077-84.

7 Anon. Consensus recommendations for the management of chronic heart failure. Am $\mathcal{F}$ Cardiol 1999;83(2A):1A-38A

8 Depre C, Taegtmeyer H. Metabolic aspects of programmed cell survival and cell death in the heart. Cardiovasc Res 2000;45:538-48.

9 Goussev A, Sharov VG, Shimoyama H, et al. Effects of ACE inhibition on cardiomyocyte apoptosis in dogs with heart failure. Am f Physiol 1998;275: H626-31.

10 Romeo F, Li D, Shi M, et al. Carvedilol prevents epinephrine-induced apoptosis in human coronary artery endothelial cells: modulation of Fas/Fas ligtosis in human coronary artery endothelial cells: modulation
and and caspase-3 pathway. Cardiovasc Res 2000;45:788-94.

11 Skudicky D, Sliwa K, Bergemann A, et al. Reduction in Fas/APO-1 plasma levels correlates with improvement in left ventricular function in patients with idiopathic dilated cardiomyopathy treated with pentoxifylline. Heart 2000;84:438.

12 Sack MN, Smith RM, Opie LH. Tumor necrosis factor in myocardial hypertrophy and ischaemia-an anti- apoptotic perspective. Cardiovasc Res 2000;45:688-95.

13 Sliwa K, Skudicky D, Candy G, et al. Randomised investigation of effects of pentoxifylline on left-ventricular performance idiopathic dilated cardiomypentoxifylline on left-ventricular per
opathy. Lancet 1998;351:1091-3.

14 Bozkurt B, Torre-Amione G, Deswal A, et al. Regression of left ventricular remodeling in chronic heart failure after treatment with ENBREL (etanercept, p75 TNF receptor Fc fusion protein) [abstract]. Circulation 1999;100:I-645

15 Vescovo G, Volterrani M, Zennaro R, et al. Apoptosis in the skeletal muscle of patients with heart failure: investigation of clinical and biochemical changes Heart 2000;84:431-7.

16 Thompson CB. Apoptosis in the pathogenesis and treatment of disease. Science 1995;267:1456-62.

17 Rose EA, Frazier OH. Resurrection after mechanical circulatory support. Circulation 1997;96:393-5.

18 Sheldon R, Connolly S, Krahn A, et al. Identification of patients most likely to benefit from implantable cardioverter-defibrillator therapy: the Canadian implantable defibrillator study. Circulation 2000;101:1660-4. 